\title{
Disability with Persistent Pain Following an Epidemic of Chikungunya in Rural South India
}

\author{
Asma A. Rahim, Romy Jose Thekkekara, Thomas Bina, and Binoy J. Paul
}

\begin{abstract}
Objective. We investigated the effects of chronic rheumatic and musculoskeletal symptoms on the functional status of people affected by the chikungunya (CKG) epidemic in the Calicut District, Kerala, South India in 2009.

Methods. A cross-sectional house-to-house survey was conducted 18 months after the CKG epidemic to assess functional status of individuals with post-epidemic persistent pain. All respondents over age 15 years with persistent pain fitting the epidemiological case definition were included. Participants' functional status was assessed using the Health Assessment Questionnaire-Disability Index (HAQ-DI). Factors affecting severity of HAQ-DI were analyzed by ordinal regression.

Results. Of 3869 subjects interviewed, 1195 (34.3\%) had a positive history of CHIKV virus infection (epidemiological or confirmed); 36.28\% (624/1720) of CKG-affected individuals had persistent pain 18 months post epidemic. Mean age of those affected was $48.22 \pm 15.6$ years; $23.2 \%$ had no disability, while $16.2 \%$ had moderate to severe disability on the HAQ-DI. Significant factors affecting severity of disability on HAQ-DI included previous rheumatic musculoskeletal disease (OR 2.27), joint and soft-tissue involvement (OR 3.74), only joint involvement (OR 2.14), female sex (OR 1.44), diet (OR 4.73), and history of joint swelling (OR 1.72).

Conclusion. Persistence of pain noted in post-CKG disease resulted in significantly deteriorated functional status of those affected. (First Release December 15 2015; J Rheumatol 2016;43:440-4; doi:10.3899/ jrheum.141609)
\end{abstract}

Key Indexing Terms: CHIKUNGUNYA FUNCTIONAL STATUS

\author{
DISABILITY \\ EPIDEMIC
}

PERSISTENT PAIN
RURAL
An epidemic of chikungunya virus (CHIKV; family Togaviridae, genus Alphavirus) spread throughout South India from 2006 onwards $^{1}$. Calicut District in North Kerala faced the brunt of this epidemic in 2008-2009. CHIKV is transmitted by both Aedes aegypti and Aedes albopictus mosquitoes. An A226V shift in the E1 gene, substitution of alanine at position 226 with valine, has led to a successful evolutionary adaptation in A. albopictus, allowing it to supplant $A$. aegypti as the primary vector ${ }^{2,3}$. The acute rheumatic manifestations of CHIKV infection typically consist of febrile arthritis. The burden of chronic manifestations of CHIKV infection remains a public health issue in affected areas. Following the reemergence of chikungunya disease (CKG) in India after 32 years, the effect of outbreaks was severe in India's southern states of Andhra Pradesh, Tamil Nadu, Karnataka, and Kerala ${ }^{4,5}$. During the 2006

From the Department of Community Medicine, Government Medical College, Kozhikode, Kerala, India.

A.A. Rahim, MD, DNB, Associate Professor; R.J. Thekkekara, Senior Research Assistant; T.B. Xavier, MD, PGDHM, Professor, Department of Community Medicine; B.J. Paul, MD, DNB, PhD, Professor, Division of Rheumatology, Department of Medicine, Government Medical College. Address correspondence to Dr. A.A. Rahim, Department of Community Medicine, Government Medical College, Kozhikode, Kerala, India 673008.E-mail:rahmaniyas@gmail.com

Accepted for publication September 11, 2015. epidemic, the estimated disability-adjusted life-years (DALY) lost in Kerala state was 1300 , with $69 \%$ due to the chronic effects of the CHIKV infection ${ }^{1}$. A phylogenetic analysis of CHIKV isolates based on partial sequences of NS4 and E1 genes from Andhra Pradesh, Karnataka, and Maharashtra indicated they belonged to the African genotype, unlike the earlier isolates that were the Asian genotype ${ }^{6}$. Post-epidemic CKG has been documented to have persistent rheumatic manifestations affecting activities of daily living $7,8,9,10,11,12$.

With a large rural population facing the consequences of the epidemic, we investigated the effects of disability from the chronic rheumatic and musculoskeletal symptoms of the postepidemic CKG on functional status.

\section{MATERIALS AND METHODS}

Site. We studied Mavoor Panchayath village in the Calicut District in North Kerala, with a population of 98,000 and which was affected by the CKG epidemic in 2009.

Sample size. Considering $52.83 \%$ of the affected group would develop persistent pain ${ }^{12}$ with $5 \%$ relative precision and $95 \%$ confidence level, a sample of $1372 \mathrm{CKG}$-affected persons was required. As revealed by a seroprevalence study, $37.2 \%$ of the population would be affected in the epidemic ${ }^{7}$; thus a population of 3880 was required with consideration of a $5 \%$ dropout rate.

Sampling frame. A cross-sectional house to house survey in the neighborhood of the village community health center was conducted 15 months after the epidemic.

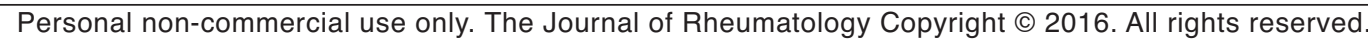


Subjects. All individuals above age 15 years with an epidemiological confirmed diagnosis of CHIKV infection and experiencing persistent pain were included in the study. The epidemiological diagnosis of CHIKV infection was made if the subject resided in or visited epidemic areas during the period of the epidemic of 2009 and had fever with severe arthralgia or arthritis $^{14}$. A positive IgM serology done during the acute phase of the infection was taken as a confirmed case. Persistent pain was defined as musculoskeletal pain that either had not remitted after the initial CHIKV fever, or that had remitted but recurred within the last 2 weeks ${ }^{7}$. The first question was, "Do you feel that you have made a complete recovery from joint manifestations since being diagnosed as having a CHIKV infection." For those who replied affirmatively, a subsidiary question was asked: "Over the past 14 days, did symptoms of CHIKV illness subside and subsequently recur?" This allowed permanently asymptomatic (remitted) patients to be differentiated from those who had experienced a relapse within the past 14 days. Patients replying negatively to the first question and affirmatively to the second were combined in a "persistence" group.

Data collection. Data were collected by trained local health volunteers to ascertain the epidemiological diagnosis of respondents using the case definition above before they were included in the study. Data included demographic characteristics, major comorbidities, and acute and chronic rheumatic manifestations and their effects on selected activities/relationships. The respondents had to subjectively grade their perceived functional impairment on these selected activities.

Tools. Anatomical sites of persistent pain were marked on a human manikin by the respondents themselves. Disability due to chronic aches and pains was determined using the Modified Indian Health Assessment Questionnaire (HAQ $)^{15,16}$, which is a version of the Stanford HAQ validated and modified for Indian use. This version was translated into the local language (Malayalam) and was pilot-tested before the study. The HAQ assessed respondents' levels of disability in 23 activities of daily living, grouped into 8 categories: arising, dressing, eating, walking, bathing, reaching, gripping, and performing errands. The level of difficulty for each activity was scored from 0 (without any difficulty) to 3 (unable to do). Finally, the highest score for activities grouped in each category was summed and divided by 8 to calculate the HAQ-DI, which ranged from 0 to 3. Based on the HAQ-DI score each patient was classified as $0=$ no difficulty, $0-1=$ mild disability, $1-1.5=$ moderate disability, and $>1.5=$ severe disability ${ }^{15,16}$.

Calculation of DALY. DALY represents the combination of both years of life lost (YLL) and years lived with disability (YLD). As no deaths attributable to CHIKV infection were seen in this community, YLL was zero. For subjects with persistent pain, YLD was calculated as a product of number of cases, a disability weight, and duration of pain. Owing to the unavailability of a disability weight for CKG disease, the disability weight for rheumatoid arthritis was used (i.e., 0.233) ${ }^{1}$. Because the duration of chronic arthralgia is known to vary over a long period of time ${ }^{1,2}$ and all our study subjects experienced pain during the interview period, 15 months was taken as the duration of pain.

Statistical analysis. Ordinal logistic regression was carried out to assess factors affecting severity of disability assessed on the HAQ-DI. Factors that were significant in univariate analysis with $\mathrm{p}$ value $<0.10$ were included in the regression analysis.

\section{RESULTS}

In total, 3869 individuals from 840 households were interviewed during the study. Of these, 1195 gave a positive history of CHIKV infection (epidemiological or confirmed); 48.6\% (95\% CI 45.79-51.45) of the CKG-affected individuals $(581 / 1195)$ had persistent pain 18 months after the epidemic. Demographic details of participants with persistent pain are given in Table 1 . We found that $85.5 \%$ of the women in the community worked at home. The majority of men in
Table 1. Demographic characteristics of subjects with persistent pain $(\mathrm{n}=$ 581).

\begin{tabular}{lc}
\hline Characteristic & Value \\
\hline Sex, \% & \\
$\quad$ Female & 62.3 \\
$\quad$ Male & 37.7 \\
Age, mean yrs (SD) & $48.22(15.60)$ \\
Religion, $\%$ & \\
$\quad$ Hindu & 66.3 \\
$\quad$ Muslim & 33.7 \\
Marital status, $\%$ & \\
$\quad$ Unmarried & 7.05 \\
$\quad$ Married & 76.95 \\
$\quad$ Widow/widower/separated & 16 \\
Years of education completed, mean (SD) & $6.21(3.7)$ \\
Diet, \% & \\
$\quad$ Vegetarian & 3.6 \\
$\quad$ Nonvegetarian & 96.4 \\
Type of family, $\%$ & \\
$\quad$ Nuclear family & 56.6 \\
Joint family & 43.4 \\
Job burden, \% & \\
$\quad$ Mild & 50.1 \\
Moderate & 37.9 \\
Severe & 12 \\
\hline
\end{tabular}

the community, $67.3 \%$, worked as manual agricultural and nonagricultural laborers. Figure 1 shows the age and sex distribution of subjects.

A history of rheumatic musculoskeletal disorder (RMSD) was noted in $53.5 \%$ of the individuals with persistent pain. Hypertension was noted in $12.4 \%$ and diabetes mellitus in $10.2 \%$ of individuals with persistent pain; $10 \%$ of the subjects were currently smokers and $5.2 \%$ consumed alcohol.

During the acute period of the CHIKV infection, in addition to fever and joint pain, $68.8 \%$ recalled joint swelling, $55.4 \%$ had rashes, $53.5 \%$ had itching, and $21.3 \%$ reported ulcers. Only $11.5 \%$ of the affected subjects took 1 to 4 weeks of rest during their initial illness. We learned that $44.2 \%$ started their work within 1 week and the remaining $44.2 \%$ started a job after more than 4 weeks.

The joints predominantly involved with persistent pain included knees (61.1\%), ankles (43.9\%), elbows (22\%), wrists (19.9\%), and shoulders (19.3\%), as shown in Table 2. Soft-tissue involvement was seen in the chest wall (25\%), leg (23.8\%), heel (17.9\%), and calf (17.6\%). We found $49.9 \%$ of the subjects had involvement of both joints and soft-tissue; $32.8 \%$ had only joint involvement and $17.3 \%$ had only soft-tissue involvement.

Persistent pain after the CHIKV infection had affected the working ability of $24.5 \%$, financial condition of $17.6 \%$, and family relations of $12.1 \%$ of the respondents. Table 3 shows the proportion of subjects with disability in the various categories of the HAQ.

There were a large proportion of subjects with mild

Personal non-commercial use only. The Journal of Rheumatology Copyright $\subset$ 2016. All rights reserved. 


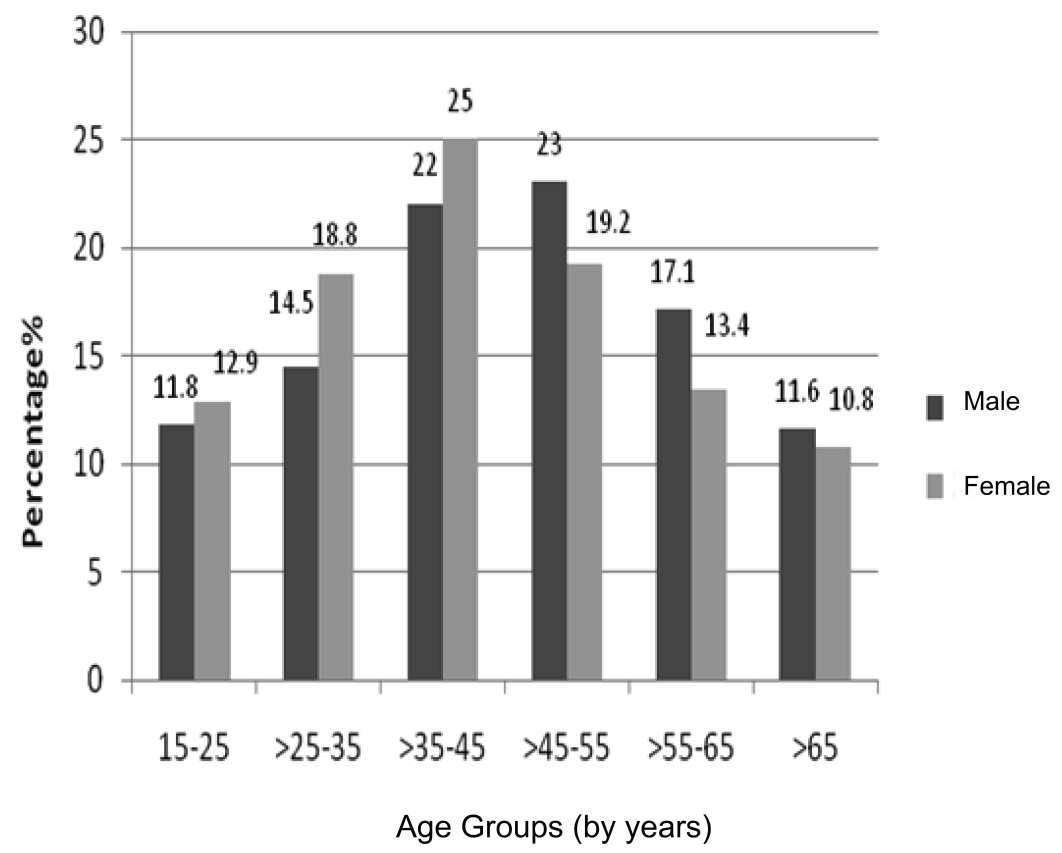

Figure 1. Data for age and sex in subjects in our study.

Table 2. Factors affecting severity of disability on the Health Assessment Questionnaire-Disability Index.

\begin{tabular}{lcc}
\hline Factors & Odds Ratio (95\% CI) & $\mathrm{p}$ \\
\hline $\begin{array}{l}\text { Female sex } \\
\text { History of rheumatic } \\
\text { musculoskeletal disorder }\end{array}$ & $1.44(1.02-2.04)$ & 0.038 \\
$\begin{array}{l}\text { History of joint swelling } \\
\quad \text { during acute disease }\end{array}$ & $2.27(1.54-3.35)$ & 0.000 \\
$\begin{array}{l}\text { Type of involvement in persistent pain } \\
\quad \text { Both joint and soft-tissue } \\
\text { involvement }\end{array}$ & 0.004 \\
$\quad \begin{array}{l}\text { Joint only } \\
\quad \text { Soft-tissue only }\end{array}$ & $3.74(2.21-6.34)$ & 0.000 \\
Vegetarian diet & $\begin{array}{c}1.14-2.49) \\
1\end{array}$ & 0.004 \\
\hline
\end{tabular}

Table 3. Percentages of subjects who experienced severe disability in activities of daily living following chikungunya disease $(n=581)$.

\begin{tabular}{lcc}
\hline Activity & Do With Much Difficulty & Unable To Do \\
\hline Arising & 20.7 & 25.1 \\
Dressing & 4 & 2.4 \\
Eating & 3.4 & 7.7 \\
Walking & 17.2 & 5.3 \\
Hygiene & 6.9 & 3.4 \\
Reaching & 9.8 & 4 \\
Gripping & 2.2 & 2.6 \\
Performing errands & 15.1 & 6.5 \\
\hline
\end{tabular}

disability $(60.6 \%) ; 23.2 \%$ of HAQ respondents had no disability, and $16.2 \%$ had moderate to severe disability on the HAQ-DI. Factors affecting severity of disability on HAQ-DI included female sex, previous RMSD, history of joint swelling during the acute disease stage, present pattern of musculoskeletal involvement, and diet (Table 2). The DALY calculated for the chronic effects of the CHIKV was 169.22 years.

\section{DISCUSSION}

The chronic rheumatic effects of CHIKV infection had an extensive impact on our study population after the epidemic. We found that the sites of involvement of joints and soft-tissue with persistent pain in our subjects were similar to sites described in other studies $7,10,11,12,17$. Although the pathogenesis of articular and nonarticular pain after CHIKV infection is not well understood, cytokines have been associated with the persistent pain ${ }^{18,19,20,21}$.

The effects of the persistent pain were felt by subjects in their family relations, working abilities, and economic conditions. The group aged 35-55 years, which forms the main working group in the community, was most significantly affected by persistent pain. As most income-earners of the families were in this age bracket, the chronic sequelae of CHIKV infection in this group adversely affected the financial conditions of their families, especially as it is a community of mainly manual labourers.

The activity categories of the HAQ in which disability was more prevalent in our study population included arising, walking, and performing errands (Table 3 ). Difficulty in these 
activities follows from the fact that the lower limbs were the most affected region, showing both soft-tissue and articular involvement. In comparison to the Bhigwan report on RMSD, in our study the disability caused by persistent pain post-CKG, in individual categories, was more severe ${ }^{11,17}$. In our study, there was a larger proportion of subjects in the "unable to do" group in 6 of the 8 individual HAQ categories, except walking and hygiene, compared to the Bhigwan study. However, on calculations of the grades of disability, a greater number of subjects in our study had no disability compared to results from the Bhigwan study. Seventy-seven percent of our cases with persistent pain post-CHIKV infection had mild to severe disability on the HAQ-DI, compared to $95 \%$ of subjects with RMSD in the Bhigwan study. Hence we may infer that although the persistent pain was not disabling to all the affected subjects, those with disability had more severe grades in the individual HAQ-DI categories. The question remains whether the aftermath of the CHIKV epidemic has brought about a change in the epidemiology of RMSD in the community. CHIKV strains with an Asian genotype of the E1 gene were reportedly detected during the 1963-1973 outbreaks in India, while more recent outbreaks, since 2005, have been caused by the Central/East African genotype. Additionally, a mutation at 226 amino acid (valine-alanine) of the $E 1$ gene was observed during the recent outbreaks, and it has been associated with the more efficient replication of CHIKV in A. albopictus ${ }^{22,23}$. Female sex was associated with greater severity of disability on the HAQ-DI. This could be attributed to the fact that women, who were mainly working in the home, would not be able to receive the adequate rest required during the convalescence period, and would resume heavy household chores before tapering of acute disease, thus predisposing them to a more severe chronic disease state ${ }^{24}$.

A history of previous RMSD increased the probability of severe disability by more than 2 times in patients with persistent pain. Biomarkers such as interleukin 6, C-X-C motif chemokine 10 (CXCL10), and CXCL9 have been found to be associated with severity of chronic CHIKV disease, and these are also expressed in patients with rheumatoid arthritis and other arthritis-related disease $e^{8,19,20,21}$. Hence an added effect of the post-CHIKV infection on previous RMSD may be precipitating a more severely disabling disease.

In the pattern of persisting pain we studied, compared to only soft-tissue involvement, both joint and soft-tissue involvement and only joint involvement increased the occurrence of a more severe disability. A vegetarian diet was also seen to increase the severity of disability. The role of diet in arthritis remains uncertain ${ }^{25,26}$. This finding must be considered with caution and awaits more detailed studies, as certain micronutrient deficiencies may have a role in arthritis ${ }^{27,28}$. The DALY lost due to persistent pain in this community were far more than the DALY lost as a result of CKG infection previously reported ${ }^{1}$. This may be due to the higher incidence of effects of CHIKV and the longer duration of chronic disease in our study community. A history of joint swelling in addition to the fever and arthralgia during the acute-disease phase points to a more severe host inflammatory response to the CHIKV infection. In turn, this initial arthritis has proven to be a predictor for recurrence of pain ${ }^{24}$. We found that the presence of joint swelling predicts not only a chronic disease but one with greater disabling capacity.

Some limitations of our study should be examined. Subjective bias on the part of respondents in grading their perceived functional impairment on the selected activities may have influenced the assessment of disability to some extent. The possibility of recall bias, leading to underestimation of current or past symptoms cannot be excluded. Serological confirmation using an IgG ELISA could not be performed in this study due to lack of facilities. A final rheumatology evaluation by clinical evaluations and investigations could not be done.

As mortality in chikungunya disease is not very common, serious effects of the disease's morbidity are often overlooked by planners and policy makers ${ }^{29}$. The social and economic burden that a CHIKV epidemic can place on a community through its chronic morbidity is profound and must be dealt with seriously. Efforts must be given to prevention of CKG disease, and in areas that have already experienced an epidemic and now deal with the effects of persistent disability, strong steps for rehabilitation through a well planned national program are necessary for affected patients to achieve complete physical, mental, and social well-being.

\section{ACKNOWLEDGMENT}

The authors thank Dr. Arvind Chopra, Coordinator, WHO-ILAR-COPCORD, and Director and Chief Rheumatologist, Centre for Rheumatic Diseases, Pune, India.

\section{REFERENCES}

1. Krishnamoorthy K, Harichandrakumar KT, Krishna Kumari A, Das LK. Burden of chikungunya in India: Estimates of disability adjusted life years (DALY) lost in 2006 epidemic. J Vector Borne Dis 2009;46:26-35.

2. Khan AH, Morita K, del Carmen Parquet M, Hasebe F, Mathenge EG, Igarashi A. Complete nucleotide sequence of chikungunya virus and evidence for an internal polyadenylation site. J Gen Virol 2002;83:3075-84

3. Vazeille M, Moutailler S, Coudrier D, Rousseaux C, Khun H, Huerre M, et al. Two Chikungunya isolates from the outbreak of La Reunion (Indian Ocean) exhibit different patterns of infection in the mosquito, Aedes albopictus. PLoS One 2007;2:e1168.

4. Santhosh SR, Dash PK, Parida M, Khan M, Rao PV. Appearance of E1:A226V mutant Chikungunya virus in coastal Karnataka, India during 2008 outbreak. Virol J 2009;6:172.

5. Kannan M, Rajendran R, Sunish IP, Balasubramaniam R, Arunachalam N, Paramasivan R, et al. A study on Chikungunya outbreak during 2007 in Kerala, South India. Indian J Med Res 2009;129:311-5.

6. Yergolkar PN, Tandale BV, Arankalle VA, Sathe PS, Sudeep A, Gandhe SS, et al. Chikungunya outbreaks caused by African genotype. India Emerg Infect Dis 2006;12:1580-3.

7. Sissoko D, Malvy D, Ezzedine K, Renault P, Moscetti F, Ledrans M,

Personal non-commercial use only. The Journal of Rheumatology Copyright $\subset$ 2016. All rights reserved. 
et al. Post-epidemic Chikungunya disease on Reunion Island: Course of rheumatic manifestations and associated factors over a 15-month period. PLoS Negl Trop Dis 2009;3:e389.

8. Chow A, Her Z, Ong EK, Chen JM, Dimatatac F, Kwek DJ, et al. Persistent arthralgia induced by Chikungunya virus infection is associated with interleukin-6 and granulocyte macrophage colony-stimulating factor. J Infect Dis 2011;203:149-57.

9. Padmakumar B, Jayan JB, Menon R, Kottarathara AJ. Clinical profile of chikungunya sequelae, association with obesity and rest during acute phase. Southeast Asian J Trop Med Public Health 2010;41:85-91.

10. Mathew AJ, Goyal V, George E, Thekkemuriyil DV. Rheumatic-musculoskeletal pain and disorders in a naive group of individuals 15 months following a Chikungunya viral epidemic in south India: A population based observational study. Int J Clin Pract 2011;65:1306-12.

11. Chopra A, Anuradha V, Ghorpade R, Saluja M. Acute Chikungunya and persistent musculoskeletal pain following the 2006 Indian epidemic: A 2-year prospective rural community study. Epidemiol Infect 2012;140:842-50.

12. de Andrade DC, Jean S, Clavelou P, Dallel R, Bouhassira D. Chronic pain associated with the chikungunya fever: Long lasting burden of an acute illness. BMC Infect Dis 2001;20:31.

13. Sissoko D, Moendandze A, Malvy D, Giry C, Ezzedine K, Solet JL, et al. Seroprevalence and risk factors of Chikungunya virus infection in Mayotte, Indian Ocean, 2005-2006: A population-based survey. PloS One 2008;3:e3066.

14. World Health Organization/South-East Asia Regional Office. Guidelines for prevention and control of Chikungunya fever. 2009. [Internet. Accessed November 9, 2015.] Available from: http://www.wpro.who.int/mvp/topics/ntd/Chikungunya_ WHO_SEARO.pdf

15. Centre for Rheumatic Diseases, Pune, India. Health Assessment Questionnaire (Modified CRD-Pune version). [Internet. Accessed November 9, 2015.] Available from: http://www.rheumatologyindia.org/pdf/HAQ.pdf

16. Chopra A, Manjit S. Validation and usefullness of Indian version (CRD Pune) Health Assessment Questionnaire: Drug trials, community practice and COPCORD Bhigwan population study (1994-2012). Ind J Rheumatol 2012;7:74-82.

17. Chopra A, Saluja M, Patil J, Tandale HS. Pain and disability, perceptions and beliefs of a rural Indian population: A WHO-ILAR COPCORD study. WHO-International League of Associations for Rheumatology. Community Oriented Program for Control of Rheumatic Diseases. J Rheumatol 2002;29:614-21.
18. Venugopalan A, Ghorpade RP, Chopra A. Cytokines in acute Chikungunya. PLoS One 2014;9:e111305.

19. Kelvin AA, Banner D, Silvi G, Moro ML, Spataro N, Gaibani P, et al. Inflammatory cytokine expression is associated with chikungunya virus resolution and symptom severity. PLoS Negl Trop Dis 2011;5:e1279.

20. Schaible HG, von Banchet GS, Boettger MK, Bräuer R, Gajda M, Richter F, et al. The role of proinflammatory cytokines in the generation and maintenance of joint pain. Ann NY Acad Sci 2010;1193:60-9.

21. Chaaitanya IK, Muruganandam N, Sundaram SG, Kawalekar O, Sugunan AP, Manimunda SP, et al. Role of proinflammatory cytokines and chemokines in chronic arthropathy in CHIKV infection. Viral Immunol 2011;24:265-71.

22. Sudeep AB, Parashar D. Chikungunya: an overview. J Biosci 2008;33:443-9.

23. Santhosh SR, Dash PK, Parida M, Khan M, Rao PV. Appearance of E1:A226V mutant Chikungunya virus in Coastal Karnataka, India during 2008 outbreak. Virol J 2009;6:172.

24. Vijayakumar KP, Anish TS, George B, Lawrence T, Muthukkutty SC, Ramachandran R. Clinical profile of chikungunya patients during the epidemic of 2007 in Kerala, India. J Global Infect Dis 2011;3:221-6.

25. Smedslund G, Byfuglien MG, Olsen SU, Hagen KB. Effectiveness and safety of dietary interventions for rheumatoid arthritis: A systematic review of randomized controlled trials. J Am Diet Assoc 2010;110:727-35.

26. Hafström I, Ringertz B, Spångberg A, von Zweigbergk L, Brannemark S, Nylander I, et al. A vegan diet free of gluten improves the signs and symptoms of rheumatoid arthritis: The effects on arthritis correlate with a reduction in antibodies to food antigens. Rheumatology 2001;40:1175-9.

27. Koller LD, Exon JH. The two faces of selenium-deficiency and toxicity are similar in animals and man. Can J Vet Res 1986; 50:297-306.

28. Judd PA, Long A, Butcher M, Caygill CP, Diplock AT. Vegetarians and vegans may be most at risk from low selenium intakes. BMJ 1997;314:1834.

29. Rahim A, Mathew AJ. Perennial impression of an emerging arbovirus on the epidemiology of rheumatic diseases in South India: Insights from the COPCORD study. J Postgrad Med 2011;57:226-8. 